\title{
Effects of induced hyperinsulinaemia with and without hyperglycaemia on measures of cardiac vagal control
}

\author{
M. Berkelaar • E. M. W. Eekhoff • A. M. C. Simonis-Bik • \\ D. I. Boomsma • M. Diamant • R. G. Ijzerman • \\ J. M. Dekker • L. M. 't Hart • E. J. C. de Geus
}

Received: 6 October 2012 / Accepted: 10 January 2013 /Published online: 13 February 2013

(C) Springer-Verlag Berlin Heidelberg 2013

\begin{abstract}
Aims/hypothesis We examined the effects of serum insulin levels on vagal control over the heart and tested the hypothesis that higher fasting insulin levels are associated with lower vagal control. We also examined whether experimentally induced increases in insulin by beta cell secretagogues, including glucagon-like peptide-1 (GLP-1), will decrease vagal control.

Methods Respiration and ECGs were recorded for 130 healthy participants undergoing clamps. Three variables of cardiac vagal effects (the root mean square of successive differences [rMSSD] in the interbeat interval of the heart rate [IBI], heart-rate variability [HRV] caused by peakvalley respiratory sinus arrhythmia [pvRSA], and highfrequency power $[\mathrm{HF}]$ ) and heart rate (HR) were obtained
\end{abstract}

M. Berkelaar · E. M. W. Eekhoff • A. M. C. Simonis-Bik •

M. Diamant $\cdot$ R. G. Ijzerman

Diabetes Center, VU University Medical Center, Amsterdam, the Netherlands

D. I. Boomsma • E. J. C. de Geus $(\bowtie)$

Department of Biological Psychology, Vrije Universiteit,

van der Boechorststraat 1,

1081 BT Amsterdam, the Netherlands

e-mail: jcn.de.geus@psy.vu.nl

\section{J. M. Dekker}

Epidemiology and Biostatistics and EMGO Institute for Health and Care Research, VU University Medical Center, Amsterdam, the Netherlands

\section{M. 't Hart}

Molecular Cell Biology, Leiden University Medical Center,

Leiden, the Netherlands

L. M. 't Hart

Section of Molecular Epidemiology, Leiden University Medical

Center, Leiden, the Netherlands at seven time points during the clamps, characterised by increasing levels of insulin (achieved by administering insulin plus glucose, glucose only, glucose and GLP-1, and glucose and GLP-1 combined with arginine).

Results Serum insulin level was positively associated with $\mathrm{HR}$ at all time points during the clamps except the firstphase hyperglycaemic clamp. Insulin levels were negatively correlated with variables of vagal control, reaching significance for rMSSD and $\log _{10} \mathrm{HF}$, but not for pvRSA, during the last four phases of the hyperglycaemic clamp (hyperglycaemic second phase, GLP-1 first and second phases, and arginine). These associations disappeared when adjusted for age, BMI and insulin sensitivity. Administration of the beta cell secretagogues GLP-1 and arginine led to a significant increase in HR, but this was not paired with a significant reduction in HRV measures.

Conclusion/interpretation Experimentally induced hyperinsulinaemia is not correlated with cardiac vagal control or HR when adjusting for age, BMI and insulin sensitivity index. Our findings suggest that exposure to a GLP-1 during hyperglycaemia leads to a small acute increase in HR but not to an acute decrease in cardiac vagal control.

Keywords Autonomic nervous system - GLP-1 - Heart rate variability $\cdot$ Insulin $\cdot$ Parasympathetic activity

$\begin{array}{ll}\text { Abbreviations } \\ \text { GLP-1 } & \text { Glucagon-like peptide-1 } \\ \text { HF } & \text { High-frequency power } \\ \text { HRV } & \text { Heart-rate variability } \\ \text { IBI } & \text { Interbeat interval of the heart rate } \\ \text { ISI } & \text { Insulin sensitivity index } \\ \text { pvRSA } & \text { Peak-valley respiratory sinus arrhythmia } \\ \text { rMSSD } & \text { Root mean square of successive differences } \\ \text { RSA } & \text { Respiratory sinus arrhythmia }\end{array}$




\section{VU-AMS Vrije Universiteit Ambulatory Monitoring System \\ VUmc VU University Medical Center}

\section{Introduction}

People with obesity and insulin resistance are characterised by decreased heart rate (HR) variability (HRV) compared with healthy controls [1-4]. Low HRV is a predictor for allcause and cardiac mortality in pre-morbid populations and in various samples of cardiac patients [5-11]. One mechanism that has been proposed to explain the risk conveyed by low HRV is a decrease in cardiac vagal control, which acts to protect against arrhythmic events $[6,8]$. A likely cause of the lower HRV in individuals with insulin resistance, often accompanied by the metabolic syndrome, is their higher levels of insulin. Several studies revealed that an acute increase in insulin decreases HRV and/or increases HR [1, 3, 12-16]. Most studies focus on sympathetic control over the heart, but there has been relatively little research on the relationship between insulin and vagal control.

To measure cardiac vagal control, various HRV measures are in use. Although all HRV measures are sensitive to changes in vagal activity, HRV in the respiratory frequency range $(0.15-0.4 \mathrm{~Hz})$, also called respiratory sinus arrhythmia (RSA), is the preferred measure of cardiac vagal control [17-20].

The aim of this study was to test the influence of stepwise increases in serum insulin level on vagal activity. Insulin levels were experimentally manipulated by exogenous insulin administration and administration of secretagoguesglucose, glucagon-like peptide-1 (GLP-1) receptor agonist and arginine-that increase insulin levels endogenously. This was achieved during a euglycaemic-hyperinsulinaemic clamp and a modified hyperglycaemic clamp with GLP-1 and arginine in healthy participants who underwent continuous recording of the ECG and respiratory signals. Because of its clinical application, we have a special interest in the effects that the beta cell secretagogue GLP-1 (in addition to high glucose and insulin levels) has on vagal control in this setting. We hypothesised that higher insulin levels are associated with lower cardiac vagal control, independently of $\mathrm{BMI}$ and insulin resistance, and that experimentally induced increases in exogenous or endogenous insulin would decrease cardiac vagal control.

\section{Methods}

Participants Between September 2004 and the end of 2006, 154 families were selected from the Netherlands Twin
Register to be invited to take part in the Dutch twin-family study 'Genetic influences on beta cell function'. The study protocol was approved by the Medical Ethics Committee of the VU University Medical Center (VUmc) and all participants gave informed consent. Only healthy participants were included, for further inclusion and exclusion criteria see Simonis-Bik et al [21]. Overall, 130 participants (100 twins and 30 siblings) participated in both a euglycaemichyperinsulinaemic clamp and a modified hyperglycaemic clamp. Three participants were excluded from the analysis because their ECG recordings were too noisy during critical parts of the experiment. The remaining 127 participants were 65 women and 62 men aged 20 to 51 years (mean 31.5 years, SD 6.3) with a BMI ranging from 18 to $36 \mathrm{~kg} / \mathrm{m}^{2}$ (mean $24.1 \mathrm{~kg} / \mathrm{m}^{2}$, SD 3.5 ).

Clamps The clamp tests were performed on a day that started at 08:00 hours in an academic research unit after a $12 \mathrm{~h}$ fast. After weight measurement (balance scale Seca; Schinkel, Nieuwegein, the Netherlands) the participant was confined to bed and an ECG/respiration recording device (Vrije Universiteit Ambulatory Monitoring System [VU-AMS], VU University, Amsterdam, the Netherlands) was attached.

The euglycaemic clamp was performed as in SimonisBik et al [21] and the modified hyperglycaemic clamp was performed as described previously in Simonis-Bik et al and Fritsche et al [21, 22]. Briefly, the euglycaemic clamp was carried out with a primed continuous insulin (Velosuline/Actrapid [Novo Nordisk, Bagsvaer, Denmark] in $\mathrm{NaCl}$ $0.9 \%$ [wt/vol.] with $2 \%$ [wt/vol.] albumin) infusion ( $40 \mathrm{mU}$ $\mathrm{m}^{-2} \mathrm{~min}^{-1}$ ) for $120 \mathrm{~min}$ and the blood glucose was kept stable at $0.3 \mathrm{mmol} / \mathrm{l}$ below the fasting level and within the range $4.5-5.5 \mathrm{mmol} / \mathrm{l}$. The hyperglycaemic clamp started with a bolus injection of glucose, and continued with glucose infusion, steady at around $10 \mathrm{mmol} / \mathrm{l}$. In addition to the glucose infusion, a bolus injection of GLP-1 (7-36 Amide Human; Polypeptide Laboratories, Wolfenbuettel, Germany), $1.5 \mathrm{pmol} \mathrm{kg}^{-1} \mathrm{~min}^{-1}$, was given and GLP-1 infusion $\left(0.5\right.$ pmol kg $\left.{ }^{-1} \mathrm{~min}^{-1}\right)$ was continued for $80 \mathrm{~min}$. Finally, a bolus injection of arginine ( $5 \mathrm{~g}$, arginine hydrochloride manufactured by VUmc pharmacists) was added in addition to the glucose and GLP-1.

Blood samples were taken frequently, with 24 withdrawals in total (represented in Fig. 1 as the small black squares just above the timeline). BP measurements were performed in duplicate at fixed intervals (four times in total; represented in Fig. 1 as black squares with a dot in the centre) with an automatic BP meter (Dinamap procare 100; KP Medical, Houten, the Netherlands).

Mean insulin levels were assessed across seven different conditions reflecting different phases of the manipulation of insulin levels (shown as black blocks in Fig. 1). 


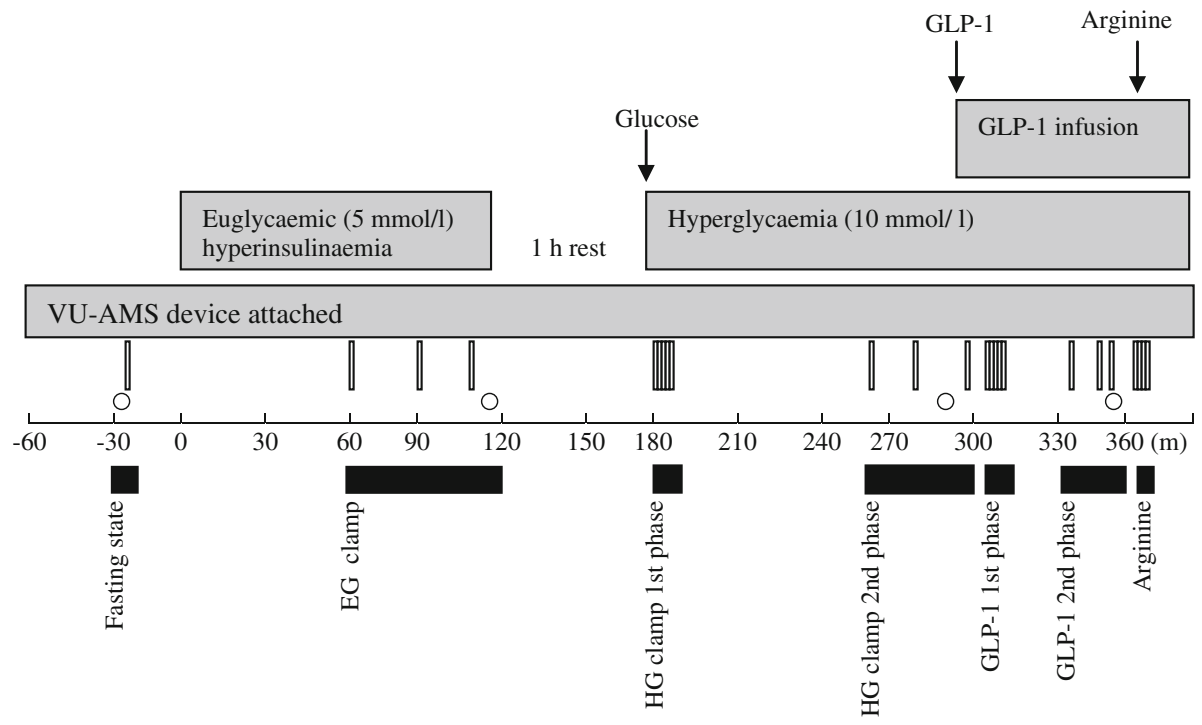

Fig. 1 Overview of the clamps: above the timeline, the grey blocks represent the different procedures; vertical bars indicate blood withdrawals; white circles indicate BP measurements; arrows indicate bolus injections. Mean steady glucose levels in the clamps are $5 \mathrm{mmol} / \mathrm{l}$ in the euglycaemic-hyperinsulinaemic clamp and $10 \mathrm{mmol} / 1$ in the hyperglycaemic clamp, as displayed in the blocks. Below the timeline, the black blocks represent the seven conditions used to test the effects of the euglycaemic-hyperinsulinaemic clamp and the modified hyperglycaemic clamp on HR and variables of cardiac vagal control. EG, euglycaemic-hyperinsulinaemic; HG, hyperglycaemic
$H R$ and HRV recording The VU-AMS device records the ECG and the thorax impedance (dZ) from six disposable, pre-gelled $\mathrm{Ag} / \mathrm{AgCl}$ electrodes as described in detail elsewhere [23-25]. Mean HR and RSA measures were assessed across the seven different conditions, as summarised in Fig. 1. Fragments of data for which participants were not quietly lying in bed (e.g. to urinate) were removed from further signal analyses.

For each of the seven experimental conditions the interbeat interval of the HR (IBI; ms) was scored using the VUAMS software suite (VU-DAMS version 2.0, VU University, Amsterdam, the Netherlands).

RSA was assessed in three ways. First, we used the 'peak-valley' method [23, 26-28]. In this method, RSA is scored from the combined respiration and IBI time series by detecting the shortest IBI during inspiration and the longest IBI during expiration on a breath-to-breath basis [23, 29]. Per breath, estimates of peak-valley RSA (pvRSA) were obtained by subtracting the shortest IBI in the inspirational phase from the longest IBI in the expiration phase. Automatic scoring of pvRSA was checked by visual inspection of the respiratory signal from the entire recording.

Second, we computed the root mean square of successive differences (rMSSD) in IBI using $\mathrm{rMSSD}$ IBI $=\sqrt{ } 1 / n \sum$ $\left(\mathrm{IBI}_{\mathrm{i}}-\mathrm{IBI}_{\mathrm{i}-1}\right)^{2}$, in which $\mathrm{IBI}_{\mathrm{i}}-\mathrm{IBI}_{\mathrm{i}-1}$ is actual IBI-previous IBI. Finally, the high-frequency power (HF) was computed as the power in the $0.15-0.40 \mathrm{~Hz}$ band, and the logarithm $\left(\log _{10} \mathrm{HF}\right)$ was determined. Mean values for
IBI, RMSSD IBI, pvRSA and $\log _{10} \mathrm{HF}$ were computed across the seven different conditions reflecting different phases of the manipulation of insulin levels. Mean IBI was converted to the more conventional notation in $\mathrm{HR}$ as $\mathrm{HR}=60,000 / \mathrm{IBI}$.

For seven participants, RSA values from a single condition were removed as outliers ( $>4$ SDs from the mean).

Statistical analyses The statistical analyses section is divided into two parts. The first part consists of the crosssectional analyses to test the association between serum insulin level and the RSA measures. In the fasting state and during the six manipulations of insulin level, zeroorder and partial Pearson product moment correlation coefficients (SPSS Statistics version 19, www-01.ibm.com/ software/analytics/spss/products/statistics/) were computed without and with the addition of the covariates age, BMI, and insulin sensitivity index (ISI) [30]. The covariates were selected based on previous findings that HRV measures differ for sex and correlate with age, BMI $[31,32]$ and ISI $[3,13]$.

The second part covers the effects of the manipulation of insulin levels. A mixed-model ANCOVA that accounts for the non-independence of family members (family = random factor) was used to test the effects of the experimental condition (fixed factor) on the RSA measures. The mixed model handles missing data in repeated measurements without removing the entire participant. Six pre-planned post-hoc tests ( $p_{\text {bonferroni }} 0.008$ ) were 
performed on the following contrasts: fasting state vs euglycaemic hyperinsulinaemic, fasting state vs hyperglycaemic second phase; fasting state vs GLP-1 second phase; fasting state vs arginine; GLP-1 first phase vs GLP-1 second phase; GLP-1 second phase vs arginine. Achieved power to detect a change of $15 \%$ of an SD in these contrasts is $0.95(n=127, \alpha 0.008)$ based on an average 0.6 correlation between the repeated measures.

\section{Results}

The means of blood insulin levels, BP, HR and the RSA measures are presented for each condition in Table 1.

Cross-sectional analyses The three measures of cardiac vagal control (HF, rMSSD IBI and pvRSA) were highly intercorrelated in the fasting state $(0.73<r<0.91)$, but the correlation was not perfect (i.e. $<1.0$ ). Fasting $\log _{10} \mathrm{HF}$ differed significantly between the sexes, with higher $\log _{10} \mathrm{HF}(2.7$ vs $2.5 ; p=0.047$ ) for women. The pvRSA, rMSSD IBI and HR were also higher for women (pvRSA 35.7 vs 33.1 ; rMSSD IBI 40.4 vs 37.7 ; and HR 67.1 vs 64.2), but this did not reach significance. Table 2 displays correlations between age, BMI and ISI with the three measurements of RSA (rMSSD IBI, $\log _{10} \mathrm{HF}$ and pvRSA), HR and insulin level. Age and BMI correlated significantly with all three measurements, whereas ISI only correlated significantly with HR. The fasting serum insulin level correlated significantly with BMI and ISI.

Table 3 shows the correlations of insulin level with HR and the three RSA measures during the entire experimental protocol. The first column depicts the observed correlations. The second column depicts the partial correlations, after correcting for the effects of age, BMI and ISI on insulin levels. In all conditions except the euglycaemic clamp,
Table 2 Correlations between covariates and variables

\begin{tabular}{|c|c|c|c|c|c|c|}
\hline \multirow[t]{2}{*}{ Variable } & \multicolumn{2}{|l|}{ Age } & \multicolumn{2}{|l|}{ BMI } & \multicolumn{2}{|l|}{ ISI } \\
\hline & $r$ & $p$ & $r$ & $p$ & $r$ & $p$ \\
\hline rMSSD IBI & -0.24 & $0.008^{* *}$ & -0.31 & $0.000^{* * *}$ & 0.15 & 0.106 \\
\hline $\log _{10} \mathrm{HF}$ & -0.34 & $0.000^{* * * *}$ & -0.35 & $0.000^{* * *}$ & 0.18 & 0.042 \\
\hline pvRSA & -0.31 & $0.001^{* * *}$ & -0.30 & $0.001^{* * *}$ & 0.06 & 0.478 \\
\hline HR & -0.11 & 0.244 & 0.14 & 0.120 & -0.33 & $0.000^{* * *}$ \\
\hline $\begin{array}{l}\text { Serum insulin } \\
\text { level }\end{array}$ & 0.04 & 0.665 & 0.54 & $0.000^{* * * *}$ & 0.52 & $0.000^{* * *}$ \\
\hline
\end{tabular}

Data are correlations (Pearson's $r$ and $p$ value) between covariates and variables (fasting state)

Significant correlations corrected for multiple comparisons $(p<0.01)$ are marked with ${ }^{* *}$ for $p<0.01$ and ${ }^{* * *}$ for $p<0.001$

higher insulin was associated with a higher HR and a lower RSA. In the analyses adjusted for age, BMI and ISI the association between HR and insulin remained partially intact, but the association between RSA measures and insulin disappeared. To explore whether the correlation between insulin level with HR and RSA exists only in normal weight persons, the tests were repeated for participants with a BMI between 20 and $25 \mathrm{~kg} / \mathrm{m}^{2}$. The results remained the same; there was no significant correlation after adjusting for age and ISI.

Effects of the manipulation of insulin levels Figure 2 displays the mean values for insulin, rMSSD IBI, HF, pvRSA and HR. Insulin, HR, rMSSD IBI and HF showed a significant main effect of the condition. At peak insulin levels the HR increased by $3.9 \mathrm{bpm}$ compared with the fasting level, and the mean rMSSD IBI, HF and pvRSA decreased by $4.5 \mathrm{~ms}$, $69.0 \mathrm{~ms}^{2}\left(\log _{10} \mathrm{HF}\right.$ decreased 0.095$)$ and $4.0 \mathrm{~ms}$, respectively. Post-hoc testing on six contrasts revealed that insulin level differences were significant in all tested contrasts. Differences

Table 1 Means and SDs

\begin{tabular}{|c|c|c|c|c|c|c|c|}
\hline Condition & $\begin{array}{l}\text { Insulin level } \\
(\mathrm{pmol} / \mathrm{l})\end{array}$ & $\begin{array}{l}\text { rMSSD IBI } \\
(\mathrm{ms})\end{array}$ & $\begin{array}{l}\log _{10} \mathrm{HF} \\
(\mathrm{ms})\end{array}$ & $\begin{array}{l}\text { pvRSA } \\
(\mathrm{ms})\end{array}$ & $\begin{array}{l}\mathrm{HR} \\
(\mathrm{bpm})\end{array}$ & $\begin{array}{l}\text { Systolic BP } \\
(\mathrm{mmHg})\end{array}$ & $\begin{array}{l}\text { Diastolic BP } \\
(\mathrm{mmHg})\end{array}$ \\
\hline Fasting state $(n=126)$ & $41(20.0)$ & $39.7(18.2)$ & $2.60(0.42)$ & $35.0(19.6)$ & $65.6(8.9)$ & $117.2(11.3)$ & $68.3(8.3)$ \\
\hline $\begin{array}{l}\text { Euglycaemic-hyperinsulinaemic clamp } \\
(n=127)\end{array}$ & $445(82.8)$ & $41.9(17.9)$ & $2.65(0.39)$ & $35.3(18.6)$ & $64.2(8.0)$ & $117.7(11.1)$ & $66.0(7.4)$ \\
\hline $\begin{array}{l}\text { Hyperglycaemic clamp, first phase } \\
(n=127)\end{array}$ & $265(170.6)$ & $41.6(19.2)$ & $2.62(0.43)$ & $32.7(19.8)$ & $62.3(8.1)$ & Not available & Not available \\
\hline $\begin{array}{l}\text { Hyperglycaemic clamp, second phase } \\
(n=127)\end{array}$ & $298(218.3)$ & $41.4(18.0)$ & $2.62(0.39)$ & $33.1(16.7)$ & $64.1(8.1)$ & $116.7(10.9)$ & $65.1(8.0)$ \\
\hline GLP-1 first phase $(n=127)$ & $428(364.8)$ & $39.1(17.3)$ & $2.57(0.41)$ & $32.0(17.7)$ & $64.1(8.4)$ & Not available & Not available \\
\hline GLP-1 second phase $(n=126)$ & $2102(1741.9)$ & $37.2(18.1)$ & $2.53(0.42)$ & $31.9(19.1)$ & $68.6(8.9)$ & $120.1(12.7)$ & $65.9(7.8)$ \\
\hline Arginine $(n=125)$ & $4775(2947.3)$ & $35.2(17.7)$ & $2.51(0.46)$ & $31.0(20.2)$ & $69.5(9.6)$ & Not available & Not available \\
\hline
\end{tabular}

Data are, for each condition, the means and SDs of serum insulin level, HR, three variables of vagal cardiac activity (RMSSD IBI, $\log _{10} \mathrm{HF}$ and RSA) and BP (systolic BP and diastolic BP) 
Table 3 Zero-order and partial correlations

\begin{tabular}{|c|c|c|}
\hline \multirow[t]{2}{*}{ Condition } & Zero-order & Partial \\
\hline & $p$ & $r$ \\
\hline
\end{tabular}

\section{Fasting state}

$\begin{array}{lrlrl}\text { rMSSD IBI } & -0.22 & 0.012 & -0.08 & 0.379 \\ \log _{10} \mathrm{HF} & -0.22 & 0.012 & -0.13 & 0.161 \\ \text { pvRSA } & -0.09 & 0.320 & 0.04 & 0.695 \\ \text { HR } & 0.37 & 0.000^{* * *} & 0.20 & 0.027\end{array}$

Euglycaemic-hyperinsulinaemic clamp

$\begin{array}{lrrrr}\text { rMSSD IBI } & -0.02 & 0.843 & 0.22 & 0.016 \\ \log _{10} \text { HF } & 0.00 & 0.968 & 0.18 & 0.048 \\ \text { pvRSA } & -0.62 & 0.838 & 0.10 & 0.291 \\ \text { HR } & 0.06 & 0.532 & -0.23 & 0.012\end{array}$

Hyperglycaemic clamp, first phase

$\begin{array}{lrlrl}\text { rMSSD IBI } & -0.24 & 0.007^{* *} & -0.13 & 0.147 \\ \log _{10} \text { HF } & -0.21 & 0.017 & -0.18 & 0.055 \\ \text { pvRSA } & -0.15 & 0.110 & -0.09 & 0.358 \\ \text { HR } & 0.23 & 0.010^{* *} & 0.09 & 0.318\end{array}$

Hyperglycaemic clamp, second phase

$\begin{array}{lrlrl}\text { rMSSD IBI } & -0.36 & 0.000^{* * *} & -0.15 & 0.106 \\ \log _{10} \mathrm{HF} & -0.35 & 0.000^{* * *} & -0.17 & 0.056 \\ \text { pvRSA } & -0.21 & 0.054 & -0.04 & 0.670 \\ \text { HR } & 0.36 & 0.000^{* * *} & 0.26 & 0.003^{* *}\end{array}$

GLP-1 first phase

$\begin{array}{lrlrl}\text { rMSSD IBI } & -0.30 & 0.001^{* * *} & -0.04 & 0.691 \\ \log _{10} \mathrm{HF} & -0.30 & 0.001^{* * *} & -0.08 & 0.383 \\ \text { pvRSA } & -0.19 & 0.036 & 0.01 & 0.892 \\ \text { HR } & 0.29 & 0.001^{* * *} & 0.17 & 0.063\end{array}$

GLP-1 second phase

$\begin{array}{lrlrl}\text { rMSSD IBI } & -0.29 & 0.001^{* * *} & -0.10 & 0.256 \\ \log _{10} \mathrm{HF} & -0.30 & 0.001^{* * *} & -0.14 & 0.117 \\ \text { pvRSA } & -0.20 & 0.030 & -0.03 & 0.775 \\ \text { HR } & 0.33 & 0.000^{* * *} & 0.23 & 0.012\end{array}$

Arginine

\begin{tabular}{lrlrl} 
rMSSD IBI & -0.35 & $0.000^{* * *}$ & -0.14 & 0.126 \\
$\log _{10} \mathrm{HF}$ & -0.36 & $0.000^{* * *}$ & -0.17 & 0.059 \\
pvRSA & -0.24 & $0.008^{* *}$ & -0.04 & 0.669 \\
HR & 0.27 & $0.003^{* *}$ & 0.14 & 0.121 \\
\hline
\end{tabular}

Data are zero order and partial correlations (Pearson's $r$ and $p$ value) of vagal variables and HR with mean insulin level in every condition

Partial correlations are controlled for BMI, age and ISI

Significant correlations corrected for multiple comparisons $(p<0.01)$ are marked with ${ }^{* *}$ for $p<0.01$ and ${ }^{* * *}$ for $p<0.001$

in HR were significant for the fasting state vs arginine (increase in HR +3.9 bpm), fasting state vs GLP-1 second phase $(+3.0 \mathrm{bpm})$, and GLP-1 first phase vs GLP-1 second phase $(+4.5 \mathrm{bpm})$ but not for fasting state vs euglycaemic hyperinsulinaemic, fasting state vs hyperglycaemic second
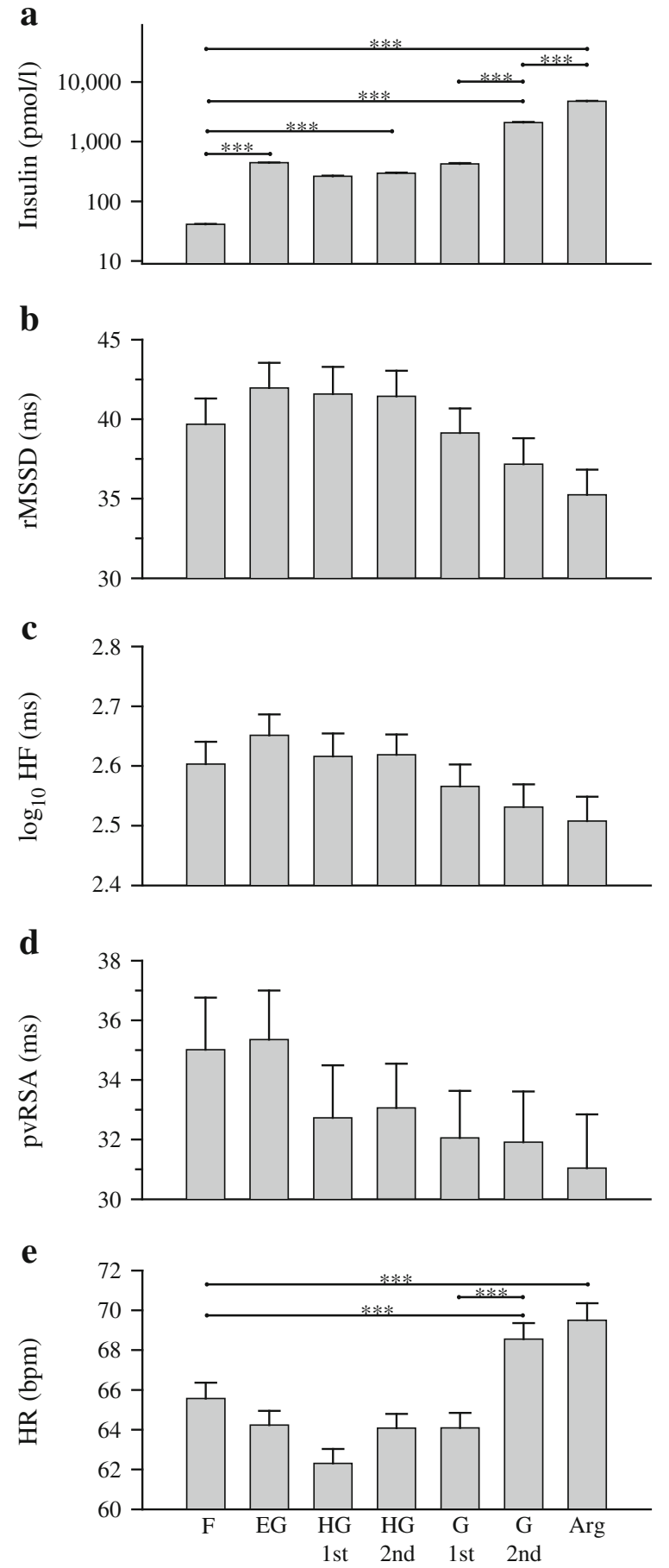

Fig. 2 Means and SEM of all participants per condition of serum insulin level, cardiac vagal control and HR. Means of all participants $(n=127)$, per condition, for serum insulin level (a), cardiac vagal control (b-d) and HR (e). Error bars show mean \pm 2 SEMs. Main effects for conditions were significant in $\mathbf{a}-\mathbf{c}$ and $\mathbf{e}(p<0.001$ by ANCOVA). Significant contrasts $\left(p_{\text {bonferroni }}<0.008\right)$ are marked with horizontal lines in (a) and (e) $(* * * p<0.001)$. There were no significant contrasts for rMSSD, HF or pvRSA. Note that the $y$-axis in (a) is a logarithmic $\left(\log _{10}\right)$ scale. F, fasting; EG, euglycaemichyperinsulinaemic; HG, hyperglycaemic; G, GLP-1; Arg, arginine; 1 st and 2 nd refer to 1 st and 2 nd phase, respectively; the $x$-axis applies to all figure parts 

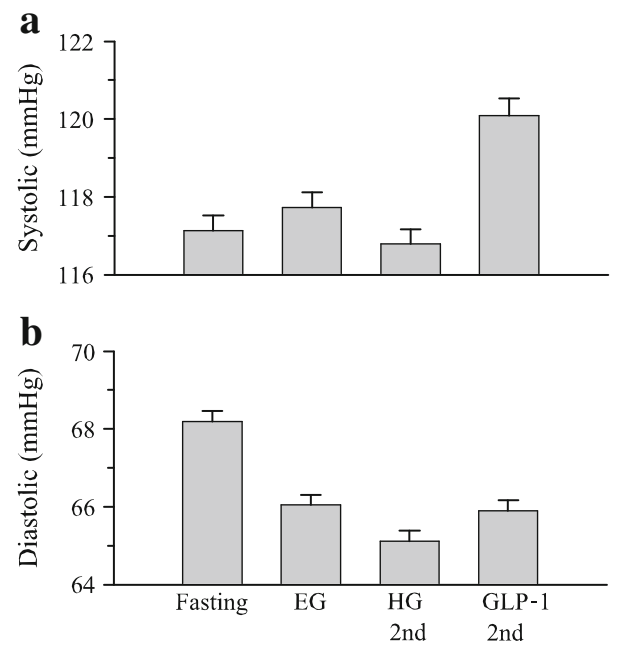

Fig. 3 Means and SEM of all participants $(n=127)$, per condition, for (a) systolic BP and (b) diastolic BP. Error bars show $\mathrm{SEM} \pm 2 \mathrm{SEM}$. EG, euglycaemic-hyperinsulinaemic; HG, hyperglycaemic; the $x$-axis applies to both figure parts

phase and GLP-1 second phase vs arginine. These posthoc contrasts were not significant for any of the RSA measurements.

Some $(n=15)$ participants felt sick, dizzy or light headed during the arginine condition. To explore whether the effects on HR were caused by nausea, the post-hoc tests with arginine were repeated after controlling for feeling sick. HR was still significantly higher in the arginine condition.

For the six previously mentioned contrasts we computed the changes in insulin and in the HR and RSA measures. Changes in insulin level were not significantly correlated with either the changes in HR or changes in any of the RSA measures (all $p$ values $>0.01$, corrected for multiple comparisons).

Figure 3 displays the means of BP measurements in four conditions. Systolic BP showed a significant main effect of condition. Mean systolic BP was $2.9 \mathrm{mmHg}$ higher in GLP1 second phase compared with the fasting state.

\section{Discussion}

The aim of this study was to learn about the effects of high levels of insulin on cardiac vagal control. We used exogenous insulin infusion and stimulation of endogenous insulin production by different combinations of bolus injections and continued infusion of glucose and other secretagogues to increase levels of insulin.

We found significant associations between insulin levels and HR as well as between insulin levels and various RSA measures that index cardiac vagal control in the fasting state and in various phases of a modified hyperglycaemic clamp. The association of insulin level with resting HR and RSA has been observed previously, amongst others by Schroeder et al [15] in a very large sample $(N=9940)$ that compared healthy individuals with individuals with diabetes and individuals with hyperinsulinaemia. However, when we adjusted for age, BMI and ISI the association between insulin and HR was strongly attenuated and the association between insulin and RSA was no longer significant. This suggests that BMI and ISI, and not insulin level, are the proximal factors influencing HR and cardiac vagal control. This idea was further corroborated by the absence of correlations between the changes in insulin to the changes in HR and RSA during manipulation of insulin levels with glucose and the B cell secretagogues GLP-1 and arginine. The higher HR observed in individuals with high insulin levels in the second phase of the hyperglycaemic clamp and the second phase of the GLP-1 infusion appears to reflect increased cardiac sympathetic control rather than decreased cardiac vagal control.

Although not significant, in the euglycaemic clamp we even see a slight increase in RSA and a slight decrease in HR, paired with a rise in serum insulin level. This is the opposite of our hypothesis and is also in contrast with findings in other studies [3, 13]. These findings could be a coincidence or reflect the different mechanism of insulin increase. The euglycaemic clamp is the only condition in which insulin was increased by exogenous infusion instead of by endogenous production by the beta cell.

Although we find no evidence of an acute effect of increased insulin production on cardiac vagal control, it is important to note that this does not rule out an effect of chronic hyperinsulinaemia on vagal control through its effects on BMI and ISI. If BMI and ISI causally affect cardiac vagal control, the effects of hyperinsulinaemia on body composition and insulin resistance indirectly create a chain of causation between high insulin levels and vagal control. From a clinical perspective, therefore, hyperinsulinaemia may still contribute to impaired vagal control in obese insulin-resistant patients with type 2 diabetes.

A limitation to our study design is that the effects of the secretagogues could not be measured separately. An example is that GLP-1 was tested in conditions of hyperglycaemia, and the true effect of GLP-1 could not be ascertained in these conditions. A second limitation is that we used a within-participant design where different stages of insulin level were induced within each participant in a predefined sequence. Thus, the effect attributed to the single conditions might also reflect the effect of a sequence of (preceding) manipulations. A third limitation is that a control group was not added. The effects of inactively lying on a bed during the day on RSA measurements are unknown and can 
influence results. Adding a control group who would have received the same infusion of an $\mathrm{NaCl}$ solution would have made the results more valid.

During the modified hyperglycaemic clamp a significant increase was observed in HR during infusion of GLP-1 and GLP-1 plus arginine. Although this could reflect the very strong increases in insulin level, our data are more compatible with a true effect of the GLP-1 (in conditions of hyperglycaemia). Of note, the additional arginine more than doubled the insulin level but scarcely increased HR over the level attained during the second phase of GLP-1 infusion only. A cardiac effect of GLP-1 is also in accordance with findings of Griffioen et al [33] that showed that central or peripheral administration of GLP-1 in rats caused increased HR and decreased cardiac vagal modulation. Our study confirms these findings in humans for HR but not for cardiac vagal control, although we also observed the strongest decline in the three RSA measures after the start of GLP-1 infusion. These findings are important in light of the increased use of GLP-1 agonists as a treatment for type 2 diabetes.

We conclude that experimentally induced hyperinsulinaemia is not correlated with HR or cardiac vagal control when adjusted for BMI and ISI. In healthy people, use of a GLP-1 agonist, in conditions of hyperglycaemia, may lead to a small acute rise in HR but does not lead to a significant decrease in cardiac vagal control. Whether longer-term exposure to GLP-1 causes an accumulative reduction in cardiac vagal control in a more vulnerable diabetic patient group is an important area for future investigation.

Acknowledgements The authors thank all participants for their cooperation.

Funding This study was supported financially by the Dutch Diabetes Research Foundation (DFN2002-00-001) and the Dutch Organization for Scientific Research (NWO-MAGW 480-04-004; NWO/SPI 56464-14192).

Duality of interest $\mathrm{M}$. Diamant is a member of the advisory boards of Abbott Diabetes Care, Eli Lilly, Merck Sharp \& Dohme (MSD), Novo Nordisk, Poxel Pharma, and is a consultant for Astra-BMS and Sanofi and speaker for Eli Lilly, MSD and Novo Nordisk. Through M. Diamant, the VUmc, Amsterdam, the Netherlands receives research grants from Amylin/Eli Lilly, MSD, Novo Nordisk and Sanofi. M. Diamant receives no personal payments in connection with the above-mentioned activities, but all payments are directly transferred to the Institutional Research Foundation. The other authors declare that there is no duality of interest associated with this manuscript.

Contribution statement EdG, DIB, MD, JMD and EMWE designed the study and supervised the project; AMCSB and RGI performed the data collection; MB, LMH and EdG performed the data analysis, and MB, EMWE and EdG wrote the paper with important input from MD, AMCSB, RGI (clinical), LMH (pathophysiology), JMD (autonomic nervous system), and DIB (statistics) on various versions of the paper. All authors approved the final version of the paper.

\section{References}

1. Liao D, Cai J, Brancati FL et al (1995) Association of vagal tone with serum insulin, glucose, and diabetes mellitus-The ARIC Study. Diabetes Res Clin Pract 30:211-221

2. Liao D, Sloan RP, Cascio WE et al (1998) Multiple metabolic syndrome is associated with lower heart rate variability. The Atherosclerosis Risk in Communities Study. Diabetes Care 21:21162122

3. Paolisso G, Manzella D, Tagliamonte MR, Rizzo MR, Gambardella A, Varricchio M (1999) Effects of different insulin infusion rates on heart rate variability in lean and obese subjects. Metabolism 48:755762

4. Rodriguez-Colon SM, Li X, Shaffer ML et al (2010) Insulin resistance and circadian rhythm of cardiac autonomic modulation. Cardiovasc Diabetol 9:85

5. Huikuri HV, Tapanainen JM, Lindgren K et al (2003) Prediction of sudden cardiac death after myocardial infarction in the betablocking era. J Am Coll Cardiol 42:652-658

6. Kleiger RE, Miller JP, Bigger JT Jr, Moss AJ (1987) Decreased heart rate variability and its association with increased mortality after acute myocardial infarction. Am J Cardiol 59:256-262

7. La Rovere MT, Bigger JT Jr, Marcus FI, Mortara A, Schwartz PJ (1998) Baroreflex sensitivity and heart-rate variability in prediction of total cardiac mortality after myocardial infarction. ATRAMI (Autonomic Tone and Reflexes After Myocardial Infarction) Investigators. Lancet 351:478-484

8. La Rovere MT, Pinna GD, Hohnloser SH et al (2001) Baroreflex sensitivity and heart rate variability in the identification of patients at risk for life-threatening arrhythmias: implications for clinical trials. Circulation 103:2072-2077

9. Nolan J, Flapan AD, Capewell S, MacDonald TM, Neilson JM, Ewing DJ (1992) Decreased cardiac parasympathetic activity in chronic heart failure and its relation to left ventricular function. $\mathrm{Br}$ Heart J 67:482-485

10. Nolan J, Batin PD, Andrews R et al (1998) Prospective study of heart rate variability and mortality in chronic heart failure: results of the United Kingdom heart failure evaluation and assessment of risk trial (UK-heart). Circulation 98:1510-1516

11. Tsuji H, Larson MG, Venditti FJ Jr et al (1996) Impact of reduced heart rate variability on risk for cardiac events. The Framingham Heart Study. Circulation 94:2850-2855

12. Berne C, Fagius J, Pollare T, Hjemdahl P (1992) The sympathetic response to euglycaemic hyperinsulinaemia. Evidence from microelectrode nerve recordings in healthy subjects. Diabetologia 35:873-879

13. Paolisso G, Manzella D, Rizzo MR et al (2000) Effects of insulin on the cardiac autonomic nervous system in insulin-resistant states. Clin Sci (Lond) 98:129-136

14. Rowe JW, Young JB, Minaker KL, Stevens AL, Pallotta J, Landsberg L (1981) Effect of insulin and glucose infusions on sympathetic nervous system activity in normal man. Diabetes 30:219-225

15. Schroeder EB, Chambless LE, Liao D et al (2005) Diabetes, glucose, insulin, and heart rate variability: the Atherosclerosis Risk in Communities (ARIC) study. Diabetes Care 28:668-674

16. Muscelli E, Emdin M, Natali A et al (1998) Autonomic and hemodynamic responses to insulin in lean and obese humans. J Clin Endocrinol Metab 83:2084-2090

17. Anonymous (1996) Heart rate variability: standards of measurement, physiological interpretation and clinical use. Task Force of the European Society of Cardiology and the North American Society of Pacing and Electrophysiology. Circulation 93:1043-1065

18. Berntson GG, Bigger JT Jr, Eckberg DL et al (1997) Heart rate variability: origins, methods, and interpretive caveats. Psychophysiology 34:623-648 
19. Martinmaki K, Rusko H, Kooistra L, Kettunen J, Saalasti S (2006) Intraindividual validation of heart rate variability indexes to measure vagal effects on hearts. Am J Physiol Heart Circ Physiol 290: H640-H647

20. Nunan D, Jakovljevic DG, Donovan G, Singleton LD, Sandercock GR, Brodie DA (2010) Resting autonomic modulations and the heart rate response to exercise. Clin Auton Res 20:213-221

21. Simonis-Bik AM, Eekhoff EM, de Moor MH et al (2009) Genetic influences on the insulin response of the beta cell to different secretagogues. Diabetologia 52:2570-2577

22. Fritsche A, Stefan N, Hardt E, Schutzenauer S, Haring H, Stumvoll M (2000) A novel hyperglycaemic clamp for characterization of islet function in humans: assessment of three different secretagogues, maximal insulin response and reproducibility. Eur J Clin Invest 30:411-418

23. de Geus EJ, Willemsen GH, Klaver CH, van Doornen LJ (1995) Ambulatory measurement of respiratory sinus arrhythmia and respiration rate. Biol Psychol 41:205-227

24. Goedhart AD, van der Sluis S, Houtveen JH, Willemsen G, de Geus EJ (2007) Comparison of time and frequency domain measures of RSA in ambulatory recordings. Psychophysiology 44:203-215

25. Willemsen GH, de Geus EJ, Klaver CH, van Doornen LJ, Carroll D (1996) Ambulatory monitoring of the impedance cardiogram. Psychophysiology 33:184-193
26. Goldberger JJ, Challapalli S, Tung R, Parker MA, Kadish AH (2001) Relationship of heart rate variability to parasympathetic effect. Circulation 103:1977-1983

27. Goldberger JJ, Ahmed MW, Parker MA, Kadish AH (1994) Dissociation of heart rate variability from parasympathetic tone. Am J Physiol 266:H2152-H2157

28. Grossman P, van Beek J, Wientjes C (1990) A comparison of three quantification methods for estimation of respiratory sinus arrhythmia. Psychophysiology 27:702-714

29. Houtveen JH, Molenaar PC (2001) Comparison between the Fourier and Wavelet methods of spectral analysis applied to stationary and nonstationary heart period data. Psychophysiology 38:729735

30. Borai A, Livingstone C, Kaddam I, Ferns G (2011) Selection of the appropriate method for the assessment of insulin resistance. BMC Med Res Meth 11:158

31. Valentini M, Parati G (2009) Variables influencing heart rate. Prog Cardiovasc Dis 52:11-19

32. Valensi P, Extramiana F, Lange $C$ et al (2011) Influence of blood glucose on heart rate and cardiac autonomic function. The DESIR study. Diabet Med 28:440-449

33. Griffioen KJ, Wan R, Okun E et al (2011) GLP-1 receptor stimulation depresses heart rate variability and inhibits neurotransmission to cardiac vagal neurons. Cardiovasc Res 89:72-78 